\title{
Black hole entropy reveals a twelfth dimension
}

\author{
Itzhak Bars* \\ TH Division, CERN, CH-1211 Geneva 23, Switzerland
}

(Received 15 October 1996)

\begin{abstract}
The Bekenstein-Hawking black hole entropy in string theory and its generalizations, as expressed in terms of charges that correspond to central extensions of the supersymmetry algebra, has more symmetries than $U$ duality. It is invariant under transformations of the charges, involving a twelfth (or thirteenth) "dimension." This is an indication that the secret theory behind string theory has a superalgebra involving Lorentz nonscalar extensions (that are not strictly central), as suggested in $S$ theory, and which could be hidden in $M$ or $F$ theories. It is suggested that the idea of spacetime is broader than usual, and that a larger "spacetime" is partially present in black holes. [S0556-2821(97)00206-3]

PACS number(s): 04.70.Dy, 11.25.Hf, 11.30.Pb
\end{abstract}

\section{INTRODUCTION}

In recent work in string theory and its extensions to a secret $M, F, S, Y, \ldots$ theory, a microscopic explanation of the Beckenstein-Hawking area-entropy law for string-related black holes is obtained by counting [1-12] the number of $D$-brane states [13]. For (nearly) extreme black holes the entropy is given in terms of the charges carried by these states [8-12]. These charges correspond to central extensions of the extended supersymmetry algebra, and it has been shown that the entropy is an invariant under $U$-duality [14] transformations applied on these charges. In particular, for four-dimensional (4D) black holes the entropy is given by the quartic invariant of $\mathrm{E}_{7(7)}$ [8] and for five-dimensional black holes it is given by the cubic invariant of $\mathrm{E}_{6(6)}$ $[9,7,10]$. In higher dimensions the entropy for nonextremal black holes has also been given as an invariant of the $U$-duality group $[12,10]$.

The entropy, which is related to just counting states, is really independent of the moduli, although it is expressed in terms of central extensions that depend on the moduli. ${ }^{1}$ This simple fact distinguishes the entropy as an ideal quantity for exploring the symmetries of the secret theory. If the under-

\footnotetext{
*On sabbatical leave from the Department of Physics and Astronomy, University of Southern California, Los Angeles, California 90089-0484.

${ }^{1}$ Define $K$ as the largest compact subgroup in the duality group $U$. The moduli are classified as the right coset $U / K$. Global $U$ transformations, applied from the left on the moduli and on quantized charges, induce moduli-dependent $K$ transformations that are applied on the supercharges as well as the maximal extensions of the superalgebra. The supercharges as well as the moduli-dependent central extensions are singlets of $U$, but transform under this induced $K$, which is continuous, since it depends on the moduli. The classification of these operators under $K$ is tabulated in [15] for various dimensions. The black hole entropy is expressed in terms of central extensions that are moduli dependent, but the moduli dependence can be stripped away under $U$ transformations since the entropy is really independent of the moduli.
}

lying secret theory has a dynamical symmetry structure, such that states or operators fall into representations of its algebra, then the entropy simply counts the multiplicity in a way consistent with the representations of the dynamical symmetry. If the secret theory is suspected to have some hidden symmetries, they should show up as invariances of the entropy. For example, the $U$-duality invariance of the entropy, for all values of the moduli, as if $U$ is continuous rather than discete, is explained through the reasoning given in this paragraph.

Furthermore, if there are suspected extra "dimensions,", the entropy should be invariant under transformations that mix the extra "dimensions" with the ten dimensions (10D) that are explicit in string theory. One of the approaches to the secret theory behind string theory is $S$ theory [16], which concentrates on the global dynamical symmetry aspects of the secret theory. $S$ theory predicts a number of symmetries, some of which are outside of $U$ duality and which involve a 12th and 13th "dimension." It is the goal of this paper to demonstrate that the entropy given below is indeed invariant under transformations that mix the hidden "dimensions" with the 10 or 11 "familiar" ones. Hence the entropy serves to show the presence of extra "dimensions" (where "dimension" is used in the sense explained in ${ }^{2}$ [16]).

Specifically, in 4D define the $N=8$ superalgebra and its central extensions in an SU(8) basis:

\footnotetext{
${ }^{2}$ As in [16], it must be emphasized that the 12th (or 13th)" dimension" is not to be understood as a naive extension of the other 11 dimensions. Rather, it is the spinor space of 12D (or projected 13D spinor space) that is relevant, with 32 Weyl-Majorana supercharges $Q_{\alpha}$ of $A$ or $B$ type. The supercharges close into $\left\{Q_{\alpha}, Q_{\beta}\right\}$ $=S_{\alpha \beta}$ (Aor $B$ types) where the $32 \times 32$ symmetric matrix $S$ contains 528 bosons. The 11 usual momenta are members of $S$, and they are on an equal footing with the 528 bosons. In this paper 12D (or 13D) is used in this sense, i.e., involving the spinors in these "dimensions" and arranging the 528 bosons into subsets consistent with 12D Lorentz transformations $\operatorname{SO}(10,2)$ (the 13th "dimension", is mixed by $A \Leftrightarrow B$ duality [16]). To emphasize this point, in this paper the word "dimension" will appear in quotes whenever appropriate.
} 


$$
\begin{gathered}
\left\{Q_{\alpha A}, Q_{\dot{\beta} \bar{B}}\right\}=\sigma_{\alpha \dot{\beta}}^{\mu} P_{\mu} \delta_{A \bar{B}}, \\
\left\{Q_{\alpha A}, Q_{\beta B}\right\}=\left(i \sigma_{2}\right)_{\alpha \beta} Z_{A B}, \quad\left\{Q_{\dot{\alpha} \bar{A}}, Q_{\dot{\beta} \bar{B}}\right\}=\left(i \sigma_{2}\right)_{\dot{\alpha} \dot{\beta}} Z_{\bar{A} \bar{B}}^{*},
\end{gathered}
$$

where $\mu=0,1,2,3$ are $\operatorname{SO}(3,1)$ vector indices, $\alpha, \dot{\alpha}=1,2$ are Weyl spinor indices, and $A, \bar{A}=1, \ldots, 8$ are $\mathbf{8 , 8} *$ indices of SU(8). Then the extremal black hole entropy $S=A_{4} / 4 G_{4}=2 \pi \sqrt{I_{4}}$ is given by the quartic invariant of $\mathrm{E}_{7(7)}[8]:$

$$
I_{4}=\operatorname{Tr}\left(Z Z^{*} Z Z^{*}\right)-\frac{1}{4}\left[\operatorname{Tr}\left(Z Z^{*}\right)\right]^{2}+4 \operatorname{Pf}(Z)+4 \operatorname{Pf}\left(Z^{*}\right),
$$

where $\operatorname{Pf}(Z)=\varepsilon^{A_{1} \cdots A_{8}} Z_{A_{1} A_{2}} Z_{A_{3} A_{4}} Z_{A_{5} A_{6}} Z_{A_{7} A_{8}} /\left(4 ! 2^{4}\right)$. In this case the central charges $Z, Z^{*}$ are antisymmetric matrices that form the representations $\mathbf{2 8 , 2 8} *$ of $\mathrm{SU}(8)$. The 56 real components of these central charges depend on 56 quantized charges that form the $\mathbf{5 6}$ of $\mathrm{E}_{7(7)}$ as well as on the 70 moduli classified as the coset of $\mathrm{E}_{7(7)} / \mathrm{SU}(8)$. Because of the invariance under $\mathrm{E}_{7(7)}$, the dependence on the 70 moduli can be stripped away, leaving only the dependence on the 56 quantized charges. On the other hand, the mass of the black hole is not an invariant under $\mathrm{E}_{7(7)}$, and therefore it does depend on the 70 moduli and 56 quantized charges. It is, of course, an invariant under SU(8) which is the explicit symmetry of the superalgebra.

Similarly, in 5D define the $N=8$ superalgebra in an $\operatorname{Sp}(8)$ basis

$$
\left\{Q_{\alpha A}, Q_{\beta B}\right\}=P_{\mu}\left(C \gamma^{\mu}\right)_{\alpha \beta} \Omega_{A B}+C_{\alpha \beta} Y_{A B},
$$

where $\mu=0,1,2,3,4$ are $\mathrm{SO}(4,1)$ vector indices, $\alpha=1,2,3,4$ are Dirac spinor indices, and $A=1, \ldots, 8$ are indices in the fundamental representation of $\operatorname{Sp}(8)$. Then the extremal black hole entropy $S=A_{5} / 4 G_{5}=2 \pi \sqrt{I_{5}}$ is given by the cubic invariant of $\mathrm{E}_{6(6)}[9,7,10]$ :

$$
I_{5}=\operatorname{Tr}(Y \Omega Y \Omega Y \Omega) .
$$

In this case $\Omega_{A B}$ is the one-component antisymmetric invariant of $\mathrm{Sp}(8)$, while $Y_{A B}$ is an antisymmetric matrix, orthogonal to $\Omega_{A B}, \operatorname{Tr}(Y \Omega)=0$, which forms the 27 of $\operatorname{Sp}(8)$. The 27 central extensions $Y_{A B}$ depend on 27 quantized charges that form the 27 of $\mathrm{E}_{6(6)}$ as well as on the 27 moduli classified as the coset $\mathrm{E}_{6(6)} / \mathrm{Sp}(8)$. In higher dimensions, as well as in dimensions 4,5 , the nearly extremal black hole entropy is also expressed in terms of the central extensions of the corresponding superalgebra $[12,10]$. Our discussion below generalizes to all cases.

The strategy in this paper is to first clarify the (broken) symmetries that involve the 12th (or 13th) "dimension," rewrite the central extensions above in a basis appropriate to these symmetries, and finally show that indeed the entropy and the mass of the black hole are invariants under transformations that include the 12th (or 13th) dimension.

One implication of our discussion is that the concept of "spacetime" is broader than usually envisaged. Through the entropy considerations discussed in this paper it is demonstrated that the spacetime superalgebra contains more than the usual spacetime momenta plus Lorentz scalar central ex- tensions which have become familiar by now. A larger space that includes Lorentz nonscalar extensions (which are not strictly central) is shown to be relevant to fully realize the minimal symmetry consistent with $12 \mathrm{D}$ (or 13D) that is present in the black hole entropy. This suggests the presence of additional moduli. The broader concept of spacetime is further discussed in the last section of this paper.

\section{MIXING ORDINARY AND EXTRA “DIMENSIONS"}

\section{A. 11th dimension}

First consider a familiar case in a framework that will generalize. From the $M$-theory point of view [17-20] the black hole entropy should reflect the presence of an 11th dimension. This manifests itself in the entropy by its invariance under transformations that mix the 11th dimension with the compactified ones. These transformations are applied on the central extensions which form multiplets.

Let us identify the symmetry when $M$ theory is toroidally compactified down to $d$ dimensions. In the superalgebra of $M$ theory, one of the central extensions is the 11th momentum. The Kaluza-Klein momenta for $c$ compactified dimensions of string theory are also viewed as $c$ central extensions of the superalgebra. The superalgebra is covariant under the rotations

$$
\mathrm{SO}(c+1)
$$

which mix the $c$ compactified dimensions of string theory with the 11th dimension of $M$ theory (note that $d+c+1=11$ ). The superalgebra has more central extensions besides the Kaluza-Klein momenta, such as winding numbers and others. All central extensions fall into multiplets of $\mathrm{SO}(c+1)$, as can be seen by starting from the 11D superalgebra with all possible central extensions [18], and then compactifying $[21,15]$. In $d=4$ the 56 central extensions form the $\mathrm{SO}(7)$ multiplets $(7+21+21+7)$. Similarly, in $d=5$ the 27 central extensions form the $\mathrm{SO}(6)$ multiplets $(6+15+6)$.

$\mathrm{SO}(c+1)$ is one of the subgroups of the $U$-duality group

$$
U \supset K \supset \mathrm{SO}(c+1),
$$

where $K$ is the largest compact subgroup of $U$. In $d=4$ one has $K=\mathrm{SU}(8)$ and $\mathrm{SO}(c+1)=\mathrm{SO}(7)$. In $d=5$ one has $K=\mathrm{Sp}(8)$ and $\mathrm{SO}(c+1)=\mathrm{SO}(6)$. The presence of the extra 11th dimension in $M$ theory can be seen in all expressions by covariance under $\mathrm{SO}(c+1)$. This is a consequence of the covariance of the superalgebra under $\mathrm{SO}(c+1)$, which in turn requires multiplets of $\mathrm{SO}(c+1)$ for all operators and all states. Therefore, the black hole entropy must be invariant under $\mathrm{SO}(c+1)$.

In fact, the black hole entropy written above is invariant under the much larger $U$ transformations; hence, it is automatically invariant under its (continuous) subgroups $K \supset \mathrm{SO}(c+1)$. This is consistent with the presence of the 11th dimension on the same footing with the other $c$ compactified dimensions. In this example $\mathrm{SO}(c+1)$ invariance is a trivial consequence of $U$ invariance. This is not true for the 12th (or 13th) "dimension," but a reasoning similar to the one given above for the 11th dimension will apply. 


\section{B. 12th "dimension"}

The compactified ( $A$ or $B$ type) superalgebra has larger isometries than $\mathrm{SO}(c+1)$. The Lorentz isometry $\mathrm{SO}(d-1,1)$ in flat spacetime is evident. ${ }^{3}$ Here we are con-

$c$ compact

+2 hidden dimensions

$\mathrm{SO}(c+1,1)$

$\downarrow$

$\left.\begin{array}{l}\mathrm{SO}(c+1), 1 \text { hidden dim. } \\ \mathrm{SO}(c) \text { is common subgroup } \\ \mathrm{SO}(c)_{L} \otimes \mathrm{SO}(c)_{R}\end{array}\right\} \rightarrow$

$\uparrow$

$T$ duality $\mathrm{SO}(c, c)$

The largest isometry that is described by a compact group is $K$. It contains not only $\mathrm{SO}(c+1)$ but also $\mathrm{SO}(c)_{L} \otimes \mathrm{SO}(c)_{R}$ [which is the maximal compact group in $\mathrm{SO}(c, c)$ of $T$ duality]. The largest isometry described by a noncompact group (also commuting with the Lorentz group) is $C \equiv \mathrm{SO}(c+1,1)$ (see below for more details). The extra spacelike 11th dimension and timelike 12th "dimension" are included in this isometry. The Lorentz group together with $C$ is contained in $\mathrm{SO}(10,2)$ which mixes all the "dimensions":

$$
\mathrm{SO}(d-1,1) \times \mathrm{SO}(c+1,1) \subset \mathrm{SO}(10,2) .
$$

For example,

$$
\begin{gathered}
d=4, \quad c=6: \quad K=\mathrm{SU}(8) \quad\left(\text { in } U=\mathrm{E}_{7(7)}\right) \\
\text { and } C=\mathrm{SO}(7,1) ; \\
d=5, \quad c=5: \quad K=U \mathrm{Sp}(8) \quad\left(\text { in } U=\mathrm{E}_{6(6)}\right) \\
\text { and } C=\mathrm{SO}(6,1) .
\end{gathered}
$$

\footnotetext{
${ }^{3}$ It has been explained (see e.g., [3]) that string BogomolniPrasad-Sommerfield (BPS) states in flat spacetime evolve into black holes in curved spacetime as the coupling becomes stronger. Therefore, the counting of black hole states is in one-to-one correspondence with the counting of BPS states in flat spacetime.
}

cerned with the isometries that commute with the Lorentz group. The structure and interrelationships among the internal symmetries and dualities are explained in $[15,16]$ through the diagram

two classifications

of generators and states: under $\mathrm{SO}(c+1,1)$ or under $K$

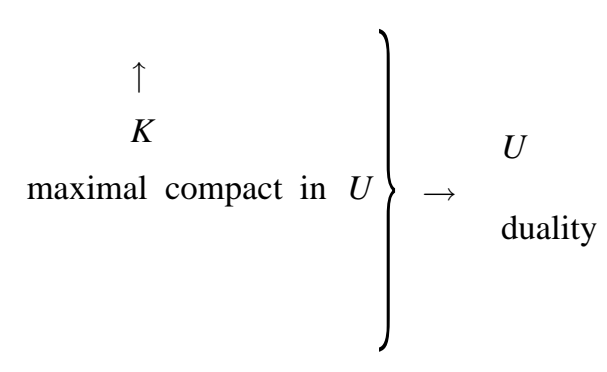

Even though $U$ is noncompact, generally it does not contain ${ }^{4}$ $C$. The essential difference between $K$ and $C$ is described as follows. For $d \leqslant 11, K$ acts as an internal symmetry group under which the $d$-dimensional momentum $P^{\mu}$ is a singlet (see below). However, under the group $\mathrm{SO}(c+1,1)$ the momentum $P^{\mu}$ is not a singlet; it mixes with other extensions that have a Lorentz index. This is related to the fact that in a 12D SO(10,2)-covariant notation the $A$-type superalgebra

$$
\begin{gathered}
\left\{Q_{\alpha}, Q_{\beta}\right\}=S_{\alpha \beta}, \\
S=\frac{1+\gamma_{13}}{2} C\left(\gamma^{M_{1} M_{2}} Z_{M_{1} M_{2}}+\gamma^{M_{1} \cdots M_{6}} Z_{M_{1} \cdots M_{6}}^{+}\right),
\end{gathered}
$$

contains no $12 \mathrm{D}$ momentum operator $P_{M}$, a property that prevents the appearance of two time coordinates or two time translation operators, labeled by $M=0,0^{\prime}[15,16]$ (the same is true for the $B$-type superalgebra). The time translation operator is $Z_{00^{\prime}}$. In compactifications to lower dimensions, the $12 \mathrm{D}$ antisymmetric tensor $Z_{M_{1} M_{2}}$ yields the momentum operator in lower dimensions in the form $P_{\mu} \equiv Z_{\mu 0^{\prime}}$. Under the transformations generated by $C=\mathrm{SO}(c+1,1)$ this momen-

\footnotetext{
${ }^{4}$ For example, in $d=8, c=2$, one has $U=\operatorname{SL}(3) \otimes \operatorname{SL}(2)$ and $C=\operatorname{SO}(3,1)$. Clearly $C$ is not contained in $U$ because it is not possible to match the compact and noncompact generators. Similarly, in $d=5$ the group $\mathrm{E}_{6(6)}$ does not contain $\mathrm{SO}(6,1)$. In some dimensions $U$ is large enough to contain a subgroup similar to $C$. For example, in $d=9, c=1$, one has $U=\operatorname{SL}(2) \otimes \operatorname{SO}(1,1)$, and $C=\operatorname{SO}(2,1)$ is isomorphic to $\operatorname{SL}(2, R)$. Similarly, in $d=4$ the group $\mathrm{E}_{7(7)}$ contains an $\mathrm{SO}(7,1)$ isomorphic to $C$. But it seems that such subgroups, when they exist, are different than $C$ since the latter acts also on the momentum $P_{\mu}$, as explained in the text.
} 
tum mixes with the other members of $Z_{\mu m}$ [defined in Eq. (12) below]. Thus, $C$ is clearly not in $U$.

The common largest compact subgroup of $K$ and $C$ is $\mathrm{SO}(c+1)$ :

$$
K \cap C=\mathrm{SO}(c+1) .
$$

The black hole entropy in every dimension $d$ is invariant under $U \supset K \supset \mathrm{SO}(c+1)$ and therefore reveals the presence of the extra 11th dimension, as discussed above. Our aim is to show that it is also invariant under $C=\mathrm{SO}(c+1,1)$ and therefore indicates the presence of a 12th "dimension" as well. As explained above this does not automatically follow from the known $U$-invariant expressions of the entropy since, generally,

$$
C \text { is not in } U \text {. }
$$

Nevertheless, the black hole entropy must be invariant under $C$. The mathematical reason behind this expectation is as follows. The (nearly) extreme black hole states are completely specified by their charges. These charges are classified by either $K$ or $C$. As indicated on the diagram above, since both $K$ and $C$ are isometries of the superalgebra of the secret theory, it must be possible to classify the operators and states under either $K$ or $C$. Completeness of states is equivalent to saying that sums of $K$ representations are expressible as sums of $C$ representations. Since the black hole entropy just counts BPS states (that fit into the short representations of the superalgebra), it must be invariant under the relabeling of the superalgebra under either $K$ or $C$. Since the counting of the total number of states cannot depend on how they are classified, the entropy must be invariant under $K$ as well as $C$.

In order to explain this symmetry we must deal with the fact that the momentum appears to be noninvariant under $C$; a new definition consistent with $C$ invariance will be introduced.

\section{CLASSIFICATION UNDER $C$}

Under compactification the $\mathrm{SO}(10,2)$ spinor $\alpha$ and vector indices $M$ of Eq. (9) are reclassified under $\mathrm{SO}(d-1,1) \otimes \mathrm{SO}(c+1,1)$

$$
\begin{gathered}
Q_{\alpha} \rightarrow Q_{\alpha a}(\text { spinor in } d \text { dims } \times \text { spinor in } c+2 \text { dims }), \\
M \rightarrow \mu(d \text { dims }) \oplus m(c+2 \text { dims }), \\
Z_{M_{1} M_{2}} \rightarrow Z_{\mu_{1} \mu_{2}} \oplus Z_{\mu_{1} m_{2}} \oplus Z_{m_{1} m_{2}},
\end{gathered}
$$$$
Z_{M_{1} \cdots M_{6}}^{+} \rightarrow \text { similar, but impose self-duality in } 12 \mathrm{D} \text {, }
$$

$$
\gamma_{M=\mu} \rightarrow \gamma_{\mu} \otimes \gamma_{c+3}, \quad \gamma_{M=m} \rightarrow \gamma_{d+1} \otimes \gamma_{m},
$$

where $\gamma_{d+1}$ and $\gamma_{c+3}$ are the analogues of $\gamma_{5}$ in 4D. This assigns the correct transformation properties under $C$ to all operators. For clarity, let us write out explicitly the general form of the superalgebra in 4D and 5D.

\section{A. 4D superalgebra}

For $d=4, c=6$, the 32 real supercharges are relabeled in the form of a complex $Q_{\alpha a}$ and $Q_{\dot{\alpha} a}=Q_{\alpha a}^{\dagger}$, where

$$
\begin{gathered}
\alpha:(1 / 2,0), \quad \dot{\alpha}:(0,1 / 2) \text { of } \operatorname{SO}(3,1), \\
a: \mathbf{8}_{+}, \quad \dot{a}: \mathbf{8}_{-} \quad \text { of } \operatorname{SO}(7,1) .
\end{gathered}
$$

Note that the $\operatorname{SO}(7,1)$ Weyl spinor indices $(a, \dot{a})$ are different than the $\mathrm{SU}(8)$ indices $(A, \bar{A})$ used in Eq. (1), although they have the same number. The maximally extended superalgebra with 528 bosonic generators takes the form

$$
\begin{gathered}
\left\{Q_{\alpha a}, Q_{\beta b}\right\}=\left(i \sigma_{2}\right)_{\alpha \beta} z_{a b}+\left(i \sigma_{2} \vec{\sigma}\right)_{\alpha \beta} \cdot \vec{F}_{a b}, \\
\left\{Q_{\dot{\alpha} a}, Q_{\dot{\beta} \dot{b}}\right\}=\left(i \sigma_{2}\right)_{\dot{\alpha} \dot{\beta}} z_{a \dot{a}}^{*}+\left(i \sigma_{2} \vec{\sigma}\right)_{\dot{\alpha} \dot{\beta}} \cdot \vec{F}_{a \dot{b}}^{*}, \\
\left\{Q_{\alpha a}, Q_{\dot{\beta} \dot{b}}\right\}=\sigma_{\alpha \dot{\beta}}^{\mu}\left(\gamma_{a \dot{b}}^{m} P_{\mu m}+\gamma_{a \dot{b}}^{m_{1} m_{2} m_{3}} A_{\mu m_{1} m_{2} m_{3}}\right) .
\end{gathered}
$$

Here the $8 \times 8 \quad \mathrm{SO}(7,1)$ Hermitian gamma matrices $\gamma^{m}=\left(1, \gamma^{k}\right)$ in Weyl spinor space are analogous to the Pauli matrix representation of the $\mathrm{SO}(3,1)$ Hermitian gamma matrices $\sigma^{\mu}=(1, \vec{\sigma})$ applied on Weyl spinors:

$$
\begin{array}{ll}
\text { SO }(3,1), \text { Weyl: } & \sigma^{\mu}=(1, \vec{\sigma}) ; \\
\text { SO(7,1), Weyl: } & \gamma^{m}=\left(1, \gamma^{k}\right) .
\end{array}
$$

The seven $\gamma^{k}$ are Hermitian, purely imaginary, and antisymmetric. The $\mathrm{SO}(3,1) \times \mathrm{SO}(7,1)$ classification of the 528 real operators on the right-hand-side and the number of their real components are

$$
\begin{gathered}
z_{a b}:\left(0,0 ; \mathbf{2 8}_{+}\right), \quad z_{a b}^{*}:(0,0 ; \mathbf{2 8}), \rightarrow 56 \text { real }, \\
\vec{F}_{a b}:(1,0 ; \mathbf{3 5}+\mathbf{1}), \quad \vec{F}_{a b}^{*}:(0,1 ; \mathbf{3 5}+\mathbf{1}), \rightarrow 216 \text { real, } \\
P_{\mu m}:\left(1 / 2,1 / 2 ; \mathbf{8}_{V}\right), \rightarrow 32 \text { real }, \\
A_{\mu m_{1} m_{2} m_{3}}:\left(1 / 2,1 / 2 ; \mathbf{5 6}_{V}\right), \rightarrow 224 \text { real. }
\end{gathered}
$$

The operators $P_{\mu m}$ are identical to the operators that come directly from the reduction from $12 \mathrm{D}$ in Eq. (12), $P_{\mu m} \equiv Z_{\mu m}$, and they include the usual momentum $P_{\mu} \equiv P_{\mu 0^{\prime}}$. The Lorentz singlet complex antisymmetric central extensions $z_{a b}, z_{a b}^{*}$, classified as the $\mathbf{2 8}_{ \pm}$of $\mathrm{SO}(7,1)$, are directly related to the two real $\mathbf{2 8}_{V}$ of $\mathrm{SO}(7,1)$ that are obtained from the reduction from $12 \mathrm{D}$ in Eq. (12):

$$
\begin{gathered}
e_{m n} \equiv Z_{m n}, \quad m_{m n} \varepsilon_{\mu_{0} \mu_{1} \mu_{3} \mu_{3}} \equiv Z_{m n \mu_{0} \mu_{1} \mu_{2} \mu_{3}}^{+}, \\
z_{a b}=\gamma_{a b}^{m n}\left(e_{m n}+i m_{m n}\right), \quad z_{a \dot{a}}^{*}=\gamma_{a \dot{a}}^{m n}\left(e_{m n}-i m_{m n}\right),
\end{gathered}
$$

where the $\gamma_{a b}^{m n}, \gamma_{a \dot{b}}^{m n}$ are antisymmetric and given by

$$
\gamma^{0^{\prime} k}= \pm i \gamma^{k}, \quad \gamma^{k l}=\frac{i}{2}\left[\gamma^{k}, \gamma^{l}\right] .
$$


These $\gamma_{a b}^{m n}, \gamma_{a \dot{a}}^{m n}$ satisfy the $\operatorname{SO}(7,1)$ Lie algebra in the $\mathbf{8}_{ \pm}$ representations. The $e_{m n}$ and $m_{m n}$ have the interpretation of "electric" and "magnetic" central charges, respectively, as can be gathered from their properties under parity (the $\varepsilon_{\mu_{0} \mu_{1} \mu_{2} \mu_{3}}$ is pseudoscalar). The black hole entropy is a function of only these Lorentz singlet central charges. Evidently, both $e_{m n}$ and $m_{m n}$ are in the adjoint ${ }^{5} \mathbf{2 8}$ of $\operatorname{SO}(7,1)$.

The remaining operators are not strictly central since they do not commute with the Lorentz generators (but they commute with each other and with the supercharges, in flat spacetime). To begin with, the Lorentz vectors $P_{\mu m}$ are not central. The Lorentz axial vectors $A_{\mu m_{1} m_{2} m_{3}}$ are related to $12 \mathrm{D}$ by

$$
A_{\mu m_{1} m_{2} m_{3}} \varepsilon_{\mu_{1} \mu_{2} \mu_{3}}^{\mu} \equiv Z_{m_{1} m_{2} m_{3} \mu_{1} \mu_{2} \mu_{3}}^{+} .
$$

The complex symmetric $\vec{F}_{a b}$ and their complex conjugates can be rewritten in the form of Lorentz-antisymmetric tensors $\left(F_{\mu \nu}\right)_{a b}$, which correspond to the $(\mathbf{1}+\mathbf{3 5})$ Lorentzantisymmetric tensors $Z_{\mu \nu}$ and $Z_{\mu \nu m_{1} m_{2} m_{3} m_{4}}^{+}$that come from the $12 \mathrm{D}$ reduction (12),

$$
\left(F_{\mu \nu}\right)_{a b} \equiv Z_{\mu \nu} \delta_{a b}+Z_{\mu \nu m_{1} m_{2} m_{3} m_{4}}^{+}\left(\gamma^{m_{1} m_{2} m_{3} m_{4}}\right)_{a b},
$$

where the $\operatorname{SO}(7,1)$ self-dual $Z_{\mu \nu m_{1} m_{2} m_{3} m_{4}}^{+}$gives the $\mathbf{3 5}$ of $\mathrm{SO}(7,1)$. Other components of $Z_{M_{1} \cdots M_{6}}^{+}$not mentioned so far are equivalent to the ones already discussed, because of its self-duality properties. This accounts for the correspondence between the 528 operators in $4 \mathrm{D}$ and 12D notation, and gives a $12 \mathrm{D}$ interpretation to the 4D superalgebra (14). This kind of reclassification of the $52812 \mathrm{D}$ type- $A$ superalgebra operators (as well as of the 13D type- $B$ superalgebra operators) in compactifications to each lower dimension was given in a table in [15].

As explained in [16], Lorentz singlet central extensions correspond to charges of point particles (or black holes), while Lorentz nonsinglet extensions correspond to boundaries of charged $p$-branes. The number of antisymmetrized Lorentz indices corresponds to $p$. Therefore, $P_{\mu m}\left(m \neq 0^{\prime}\right)$ and $A_{\mu m_{1} m_{2} m_{3}}$ correspond to end points of strings in $4 \mathrm{D}$, and $\vec{F}_{a b}$ correspond to boundaries of membranes in 4D:

$$
\begin{gathered}
\text { points } \rightarrow z_{a b}, z_{a b}^{*}, \\
\text { strings } \rightarrow P_{\mu m}\left(m \neq 0^{\prime}\right), \quad A_{\mu m_{1} m_{2} m_{3}}, \\
\text { membranes } \rightarrow \vec{F}_{a b}, \vec{F}_{a b}^{*} .
\end{gathered}
$$

If all the 528 operators in the superalgebra (14) are kept, there really is a full $\mathrm{SO}(10,2)$ isometry in disguise, since this is equivalent to the superalgebra (9). In this paper we are

\footnotetext{
${ }^{5}$ Note also that these are not the same as the $q_{i j}, p^{i j}$ defined in other references (e.g., [8]) since those are in an $\mathrm{SO}(8)$ basis rather than the $\operatorname{SO}(7,1)$ basis used here. The relation between the two is not trivial and can be obtained from the following discussion.
}

interested in describing just the black hole sector for which the algebra is evaluated on states that satisfy ${ }^{6}$

$$
\text { black hole sector: } \vec{F}_{a b}=\vec{F}_{a b}^{*}=A_{\mu m_{1} m_{2} m_{3}}=0 \text {. }
$$

So far the superalgebra simplifies to

$$
\begin{gathered}
\left\{Q_{\alpha a}, Q_{\beta b}\right\}=\left(i \sigma_{2}\right)_{\alpha \beta} z_{a b}, \\
\left\{Q_{\alpha \dot{ }}, Q_{\dot{\beta} \dot{b}}\right\}=\left(i \sigma_{2}\right)_{\alpha \beta} z_{a \dot{a}}^{*}, \\
\left\{Q_{\alpha a}, Q_{\dot{\beta} \dot{b}}\right\}=\sigma_{\alpha \dot{\beta}}^{\mu} \gamma_{a \dot{b}}^{m} P_{\mu m} .
\end{gathered}
$$

Although the full $\mathrm{SO}(10,2)$ isometry is lost by concentrating on only this sector, there still is an $\mathrm{SO}(3,1) \times \mathrm{SO}(7,1)$ isometry that has information on the 12th dimension through $\mathrm{SO}(7,1)$ that rotates the 12 th dimension into the other compactified dimensions, including the 11th. This superalgebra still allows the presence of strings.

If we also set $P_{\mu m}\left(m \neq 0^{\prime}\right)$ equal to zero to eliminate the charged black strings, then using Eq. (16), $\gamma_{a \dot{b}}^{0^{\prime}}=\delta_{a \dot{b}}$, for Weyl spinors, we obtain the standard supersymmetry algebra with the usual Lorentz singlet extensions, consistent with previous discussions of black holes. However, this further restriction on the $P_{\mu m}$ breaks the $\mathrm{SO}(7,1)$ isometry. Instead, we need to restrict the superalgebra to the black hole sector consistently with $\mathrm{SO}(7,1)$ covariance, which is important to keep track of the 12th dimension. Such a covariant condition is

$$
P_{\mu m}=P_{\mu} v_{m}
$$

where $P_{\mu}$ plays the role of $4 \mathrm{D}$ momentum and $v_{m}$ plays the role of $8 \mathrm{D}$ "momentum" in the compactified directions. Now, since there is $\mathrm{SO}(7,1)$ covariance, it is possible to apply a boost to rotate $v_{m}$ to the form $v_{m}=(1,0, \ldots, 0)$, to work in a $\mathrm{SO}(7,1)$ "rest frame" in which the superalgebra takes the standard form $\left\{Q_{\alpha a}, Q_{\dot{\beta} \dot{b}}\right\}=\sigma_{\alpha \dot{\beta}}^{\mu} \delta_{a \dot{b}} P_{\mu}$. Of course, this could be done for one given eigenvalue of the operator $v_{m}$; it cannot be done simultaneously for all the states of the secret theory, unless the whole theory is restricted to a single eigenvalue of the operator $v_{m}$. More generally, in the secret theory, we will allow all eigenvalues of $v_{m}$ just as we allow all eigenvalues of $P_{\mu}$. Evidently, now there is a parallel between the $4 \mathrm{D}$ and $8 \mathrm{D}$ sectors.

\section{Map between $S O(7,1)$ and $S U(8)$ classifications}

If one uses the product form (31), one cannot see the classification of the operators under $K \subset U$. The SU(8) structure of Eq. (1) is not evident in the $\mathrm{SO}(7,1)$-covariant form

$$
\left\{Q_{\alpha a}, Q_{\dot{\beta} \dot{b}}\right\}=\sigma_{\alpha \dot{\beta}}^{\mu} P_{\mu} \gamma_{a \dot{b}}^{m} v_{m} .
$$

An SU(8) classification is not possible unless $v_{m}$ is boosted with an $\mathrm{SO}(7,1)$ transformation to the "rest frame" $v_{m}=(1,0, \ldots, 0)$ (we have normalized $v^{m} v_{m}=1$ by absorbing the overall scale into $P_{\mu}$ ). This kind of phenomenon has been noticed before in another context: In order to display

\footnotetext{
${ }^{6} \mathrm{All}$ bosonic operators commute in flat spacetime [16]; therefore, they can be simultaneously diagonalized.
} 
electric-magnetic duality as a symmetry, one has to give up a Lorentz-covariant formalism. Similarly, in the present case, in order to display a form consistent with $\mathrm{SU}(8)$ (i.e., duality) we have to give up an $\mathrm{SO}(7,1)$-covariant formalism or vice versa.

This remark leads to the map $T$ between the $\mathrm{SU}(8)$ basis and the $\mathrm{SO}(7,1)$ spinor basis. Consider a map $T$ that relates the two bases

$$
Q_{\alpha A}=T_{A}^{a} Q_{\alpha a}, \quad Q_{\dot{\alpha} \bar{A}}=T_{\bar{A}}^{a} Q_{\dot{\alpha} a}, \quad T_{\bar{A}}^{a}=\left(T_{A}^{a}\right)^{*},
$$

where $A=1, \ldots, 8$ denotes the $\mathbf{8}$ of $\mathrm{SU}(8)$. When applied to Eq. (32) it gives the desired $\mathrm{SU}(8)$-covariant result

$\left\{Q_{\alpha A}, Q_{\dot{\beta} \bar{B}}\right\}=\sigma_{\alpha \dot{\beta}}^{\mu} P_{\mu} \delta_{A \bar{B}} \Leftrightarrow\left[T\left(\gamma^{m} v_{m}\right) T^{\dagger}\right]_{A \bar{B}}=\delta_{A \bar{B}}$.

$T_{A}^{a}(v)$ is just the $\operatorname{SO}(7,1)$ boost (in the spinor representation) and it can be constructed explicitly

$$
T=\frac{1+v_{0^{\prime}}-\vec{\gamma} \cdot \vec{v}}{\sqrt{2+2 v_{0^{\prime}}}}=T^{\dagger}, \quad T^{-1}=T^{T}=T^{*}
$$

Here $\vec{\gamma} \cdot \vec{v}$ contains only the seven Hermitian purely imaginary gamma matrices. $T^{-1}$ is just the square root of the $8 \mathrm{D}$ "momentum" matrix $\gamma^{m} v_{m}$ since it gives

$$
\left(T^{-1}\right)^{2}=v_{0}+\vec{\gamma} \cdot \vec{v}=\gamma^{m} v_{m} \quad \text { with } v_{0^{\prime}}^{2}-\vec{v}^{2}=1
$$

The SU(8) basis central extensions that appear in Eq. (1) are now written as

$$
Z_{A B}=\left(T z T^{T}\right)_{A B}, \quad Z_{\bar{A} B}^{*}=\left(T^{*} z^{*} T^{T *}\right)_{\bar{A} \bar{B}}=T^{T} z^{*} T
$$

Hence the central extensions $z_{a b}, Z_{A B}$ are functions of not only the moduli $\mathrm{E}_{7(7)} / \mathrm{SU}(8)$ but also of the additional moduli $v_{m} \sim \mathrm{SO}(c+1,1) / \mathrm{SO}(c+1)$. We will use this fact in $\mathrm{Sec}$ IV in order to show the invariance of the entropy.

\section{B. Superalgebra in 5D}

The story in 5D is similar, but has an additional important point which is not present in the 4D superalgebra. This consists of one additional Lorentz scalar central extension that is needed for $\mathrm{SO}(c+1,1)=\mathrm{SO}(6,1)$ covariance, but which is absent in the $K=\mathrm{Sp}(8)$ basis used in Eqs. (3) and (4). Understanding this point brings more clarity to the $12 \mathrm{D}$ point of view. In the remaining dimensions there are no new issues; hence, they will not be discussed here.

Following the same strategy that gave Eqs. (30) and (31), we obtain the $d=5, c=5$ superalgebra by compactification from $12 \mathrm{D}$, keeping only the relevant terms for the black hole sector:

$$
\begin{gathered}
\left\{Q_{\alpha a}, Q_{\beta b}\right\}=\left(C \gamma^{\mu}\right)_{\alpha \beta}\left(\gamma^{m}\right)_{a b} P_{\mu m}+C_{\alpha \beta} y_{a b}, \\
P_{\mu m}=P_{\mu} v_{m}, \quad y_{a b}=\left(e_{m n} \gamma^{m n}+m_{m} \gamma^{m}\right)_{a b},
\end{gathered}
$$

where $a$ denotes the eight-spinor index of $\mathrm{SO}(6,1)$ and $m=0^{\prime}, 1, \ldots, 6$ is its vector index. The seven gamma matri- ces $\gamma_{a b}^{m}$ are antisymmetric. ${ }^{7}$ The $21 \gamma^{m n}$ are also antisymmetric matrices and form the $\mathrm{SO}(6,1)$ Lie algebra. The relation of the "electric" and "magnetic" central extensions $e_{m n}, m_{m}$ to the 12D tensors in Eq. (9) is given by

$$
e_{m n} \equiv Z_{m n}, \quad m_{m} \varepsilon_{\mu_{0} \mu_{1} \cdots \mu_{4}}=Z_{m \mu_{0} \mu_{1} \cdots \mu_{4}}^{+} .
$$

The new issue is that the $\operatorname{SO}(6,1)$-covariant form (38) requires $7+21=28$ central extensions, while the $\operatorname{Sp}(8)$ covariant form (3) has only 27 of them. Although the structure of the superalgebra permits one more central extension that is an $\operatorname{Sp}(8)$ singlet, this central extension does not appear in the known 5D supergravity theories that come from compactifications of 11D supergravity (see, e.g., [22]).

To relate the $\mathrm{SO}(6,1)$ spinor basis to the $\mathrm{Sp}(8)$ basis we may again construct the transformation that corresponds to a $\mathrm{SO}(6,1)$ boost:

$$
\begin{gathered}
Q_{\alpha A}=T_{A}^{a} Q_{\alpha a}, \quad T(v \cdot \gamma) T^{T}=\Omega \equiv \gamma^{0^{\prime}}, \\
T=\frac{1+v_{0^{\prime}}-\gamma_{0^{\prime}} \vec{\gamma} \cdot \vec{v}}{\sqrt{2+2 v_{0^{\prime}}}}, \quad T^{-1}=T^{T} .
\end{gathered}
$$

Then the relation between the central extensions in the two bases is

$$
y=T^{T} Y T=e_{m n} \gamma^{m n}+m_{m} \gamma^{m}
$$

Since $Y_{A B}$ is orthogonal to $\Omega_{A B}$, this implies that

$$
m \cdot v=0 .
$$

This condition is $\mathrm{SO}(6,1)$ invariant, and it can be imposed only in the presence of the additional quantum numbers $v_{m}$ (perhaps this last restriction may be removed in a more general supergravity theory of the type suggested in the last section).

We now see that the 5D superalgebra can be rewritten covariantly under $\mathrm{SO}(6,1)$ and that the central extensions depend not only on the moduli $\mathrm{E}_{6(6)} / \mathrm{Sp}(8)$ but also on the moduli $v_{m}$ described by $\mathrm{SO}(c+1,1) / \mathrm{SO}(c+1)$. The additional moduli play a crucial role in understanding the invariance of the entropy and of the hidden 12th dimension.

\section{INVARIANCE OF THE MASS AND ENTROPY}

We are now ready to consider the extreme black holes. These correspond to short representations of the superalgebra since they satisfy the BPS conditions (the discussion is similar for the nearly extreme ones). In $S$-theory language this corresponds to requiring that the determinant of the $32 \times 32$ matrix on the right-hand side of the 12D superalgebra (9) vanish on such states

$$
\operatorname{det}(S)|\mathrm{BPS}\rangle=0
$$

\footnotetext{
${ }^{7}$ The seven $8 \times 8 \quad \gamma$ matrices that appear in Eq. (16) have been relabeled as $m=0^{\prime}, 1, \ldots, 6$ and by multiplying one of them with $i$. Note that $\gamma^{0^{\prime}}$ is not proportional to 1 in the $\operatorname{SO}(6,1)$ Dirac spinor space.
} 
This condition is equivalent to the vanishing of some linear combination of supercharges on the BPS states. This gives short multiplets of the superalgebra, corresponding to the BPS states. If the multiplicity of the zero eigenvalue of $S$ is $2 n$, then the size of the short supermultiplet is

$$
\left(2_{B}^{15-n}+2_{F}^{15-n}\right) \times \operatorname{dim} R,
$$

where $\left(2_{B}^{15-n}+2_{F}^{15-n}\right)$ corresponds to the number of independent bosonic $(B)$ or fermionic $(F)$ terms in the most general polynomial constructed with the supergenerators, in the sector with $2 n$ zero eigenvalues.

The factor $\operatorname{dim}(R)$ corresponds to the (collection of) representations $R$ of the reference state on which the supergenerators are applied. These must correspond to a representation of the isometries of the algebra, which we will call the "little group" in analogy to the little group in the representation theory of the (super-)Poincare group. The little group in our case includes either $K$ or $C$. The specific representations $R$ that are relevant depend on the details of the secret theory, but one can try to extract this information from one of its limits, such as string theory and its generalizations (including $D$-branes), by combining that information with consistency with the little group.

This representation theory method of counting states [21] will not be applied in the present paper. Instead, we will use the already known results given in the Introduction and concentrate on the additional invariances that are expected to be present under the little group, because of the procedure outlined above. By showing that the entropy, computed with other methods, is invariant under the little group, we build up evidence that the global structure of $S$ theory (together with its implied extra dimensions) is correct.

The condition $\operatorname{det}(S)=0$ is invariant under all of the isometries, $K$ or $C$ in every dimension, applied on $S$. In the black hole sector of Eqs. (29) and (31) there still is $K$ or $C$ isometry depending on the $\mathrm{SO}(c+1,1)$ frame chosen. Here $\operatorname{det}(S)$ remains invariant under the boost transformation $T$ that relates the two bases. Therefore, one has the same set of solutions $|\mathrm{BPS}\rangle$ in either the $K$ or $C$ bases, but the states are regrouped differently into a collection of representations $R$ of either $K$ or $C$. That is, the representations $R$ are the same collection of states, which can be expanded as either representations of $K$ or of $C$. Since the counting of the states, $\operatorname{dim} R$, is the same in any frame, it must be true that the entropy is invariant under both $K$ and $C$.

In the general $\mathrm{SO}(c+1,1)$ frame the determinant condition takes the form

$$
\begin{gathered}
\text { 4D: } \quad \operatorname{det}\left(\begin{array}{cc}
i \sigma_{2} \otimes z & \sigma^{\mu} P_{\mu} \otimes \gamma^{m} v_{m} \\
\sigma^{T \mu} P_{\mu} \otimes \gamma^{T m} v_{m} & i \sigma_{2} \otimes z^{*}
\end{array}\right)=0 ; \\
\text { 5D: } \quad \operatorname{det}\left(C \gamma^{\mu} P_{\mu} \otimes \gamma^{m} v_{m}+C \otimes y\right)=0 .
\end{gathered}
$$

These can be simplified (the normalization $v^{2}=v_{0}^{2}-\vec{v}^{2}=1$ is used without loss of generality by absorbing the norm into the definition of $P_{\mu}$ ):

$$
\begin{aligned}
& \text { 4D: } \quad\left[\operatorname{det}\left(P^{2} 1_{8}-v z v^{*} z^{*}\right)\right]^{2}=0, \\
& \text { 5D: } \quad\left[\operatorname{det}\left(P^{2} 1_{8}-v y v y\right)\right]^{2}=0 .
\end{aligned}
$$

Therefore in $4 \mathrm{D}$ (or 5D) the black hole mass $P^{2}=M^{2}$ is given by the largest eigenvalue of the $8 \times 8$ matrix $v z v^{T} z^{*}$ (or vyvy). The eigenvalues are obviously $\mathrm{SO}(c+1,1)$ invariants. By using this invariance we can apply a boost to go to the frame $v_{m} \rightarrow(1, \overrightarrow{0})$ to show that in $4 \mathrm{D}$ (or $5 \mathrm{D}$ ) the eigenvalue is the same as the one obtained by diagonalizing $\left(Z Z^{*}\right)_{A \bar{B}}$ in the $\mathrm{SU}(8)$ basis [or $(Y \Omega Y \Omega)_{A B}$ in the $\mathrm{Sp}(8)$ basis]. Therefore, as expected, the mass is both $C$ as well $K$ invariant.

Turning to the entropy, we use the map $T^{-1}$ in Eqs. (37) and (42) from the $K$ basis to the $C$ basis, and recall Eq. (36), $\left(T^{-1}\right)^{2}=v$, to obtain new expressions for the entropy in $4 \mathrm{D}$ and $5 \mathrm{D}$, in terms of the invariants

$$
\begin{gathered}
I_{4}=\operatorname{Tr}\left(v z v^{*} z^{*} v z v^{*} z^{*}\right)-\frac{1}{4}\left[\operatorname{Tr}\left(v z v^{*} z^{*}\right)\right]^{2} \\
+4 \operatorname{Pf}(z)+4 \operatorname{Pf}\left(z^{*}\right) \\
I_{5}=\operatorname{Tr}(v y v y v y) .
\end{gathered}
$$

These are equivalent to the ones given in the Introduction, but are now written explicitly as invariants of $\mathrm{SO}(c+1,1)$ that mix the 12th (or 13th) dimension with the other compactified dimensions. In the "rest frame" $v=(1, \overrightarrow{0})$ the new form reduces to the form given in the Introduction, after using $\gamma^{0^{\prime}}=1$ for $\operatorname{SO}(7,1)$ or $\gamma^{0^{\prime}}=\Omega$ for $\operatorname{SO}(6,1)$. The entropy really depends only on the quantized quantum numbers, but they are written in terms of central extensions that depend not only on the moduli $U / K$ but also on the moduli $v_{m} \sim \mathrm{SO}(c+1,1) / \mathrm{SO}(c+1)$.

\section{FUTURE DIRECTIONS}

We have seen that a 12D interpretation of the black hole entropy is possible. This provides some evidence for the existence of a 12th dimension that is apparently hidden in string theory black holes. It must be emphasized that the discussion was given for a 12D description of the $A$-type supersymmetry. By $T$ duality in $S$ theory, going over to the $B$-type superalgebra, this is equivalent to finding the 13th dimension in the black hole entropy.

There are several directions for expanding on the remarks made in this paper.

(1) We have seen that the superalgebra is covariant under either $K$ or $C$, but it cannot be covariant simultaneously under both. This should have been expected by analogy to the incompatibility of Lorentz covariance with electricmagnetic duality. It is known that these cannot be displayed as simultaneous covariances of the equations. In the present case $K$ is related to dualities and $C$ to Lorentz transformations. However, one quantity, the entropy, is invariant under both $C$ and $U$. Since the former is generally not fully included in $U$ (see footnote 6), except for the subgroup $\mathrm{SO}(c+1)$, it must be that there is a larger group of symmetries $G$ that leave the entropy invariant, such that

$$
\begin{gathered}
G \supseteq U \supset K \quad \text { and } \quad G \supset \mathrm{SO}(c+1,1), \\
K \supset \mathrm{SO}(c+1) \subset \mathrm{SO}(c+1,1) .
\end{gathered}
$$


This group, which includes duality transformations as well as rotations into the hidden dimensions, is likely to provide valuable information about the secret theory. Work in progress on this point will be reported elsewhere.

(2) In order to have a covariant description including the 12th "dimension" we needed the form $P_{\mu m}=P_{\mu} v_{m}$ of Eq. (31), which implies the existence of extensions $P_{\mu m}(m$ $\left.\neq 0^{\prime}\right)$ in the superalgebra that are Lorentz nonsinglets. As already emphasized, this requires the presence of open strings and, hence, the presence of nonlocality which is natural in a theory of extended objects.

Another way of understanding the nonlocality is as follows. In the sector in which $P_{\mu m}=P_{\mu} v_{m}$ the states are labeled by the eigenvalues of both operators $\left|P_{\mu}, v_{m}\right\rangle$. In a field theory description one will need to introduce bilocal fields in the Fourier space $\Phi\left(x_{\mu}, y_{m}\right)$, consistent with nonlocality. The Kaluza-Klein expansion in the $y$ variable gives a tower of local fields $\Phi_{v_{m}}\left(x_{\mu}\right)$ labeled by the eigenvalues of $v_{m}$, all of which have degenerate mass $P^{2} v^{2}=M^{2}$. We have seen that the black hole entropy and mass is consistent with the extension of spacetime in this sense.

"Spacetime" begins to have a new meaning, and we begin to enter an unknown territory.

(3) More generally, according to $S$ theory, in the presence of open $p$-branes, the superalgebra should include a larger set of Lorentz nonsinglet extensions. In their presence the concept of spacetime is expanded even further than above. In this context, our usual spacetime, as represented by the usual momentum $P_{\mu}$ in the superalgebra, does not seem to be more special as compared to the other extensions. In our current level of understanding the new parts of "spacetime" are interpreted as open $p$-branes. In the future we may find that the central extensions suggested in $S$ theory may take a more democratic role as parts of an expanded, much richer, "spacetime."

(4) It would be interesting to construct models of extended objects that fit this description of spacetime. One possibility is to consider their low energy effective theory in which one keeps some of their degrees of freedom, as in the example of a bilocal field above. A guiding principle is that the physical states should fall into a representation of the superalgebra $\{Q, Q\}=S$, with $\operatorname{det} S=0$, which is a condition for representations that correspond to both massless and massive BPS states.
Such an example in 12D was given in [16] in the form $S_{\alpha \beta}=\gamma_{\alpha \beta}^{\mu \nu}\left(p_{\mu} v_{\nu}-p_{\nu} v_{\mu}\right)$, for which the determinant condition becomes $p^{2} v^{2}-(p \cdot v)^{2}=0$. In this case $S$ has 16 zero eigenvalues; therefore, according to Eq. (45), the number of states is $2_{B}^{7}+2_{F}^{7}$ for the smallest multiplet. This representation is obviously covariant under $\mathrm{SO}(10,2)$ by construction, but yet it contains a set of fields that is identical to the one in 11D supergravity. Furthermore, in the present case the fields are bilocal $\Phi\left(x_{\mu}, y_{\nu}\right)$ and the variables are 12 dimensional with signature $(10,2)$. This suggests that a $12 \mathrm{D}$ supergravity that corresponds to this representation should exist. A plan for constructing it could include using the old gauging principles that were applied in the construction of the usual supergravity. The global algebra that provides the starting point is the superalgebra of $S$ theory.

The connection to the usual 11D supergravity can be seen as follows. The determinant constraint can be satisfied by taking equations of motion that correspond to $p^{2}=0$, $p \cdot v=0$ such as

$$
\partial_{x}^{2} \Phi\left(x_{\mu}, y_{\nu}\right)+\cdots=0, \quad \partial_{x} \cdot \partial_{y} \Phi\left(x_{\mu}, y_{\nu}\right)+\cdots=0,
$$

where the ellipses represent nonlinear interactions. If one takes a Kaluza-Klein expansion in the $y$ variable, one has the tower $\Phi_{v_{\mu}}\left(x_{\mu}\right)$ labeled by $v_{\mu}$. By keeping only one of these eigenvalues, e.g., $v_{\mu}=(1,0, \ldots, 0)$, the equations would reduce identically to $11 \mathrm{D}$ supergravity.

(5) It is worthwhile to try to build $12 \mathrm{D}$ models as the candidates for the low energy physics of the secret theory and to shed more light on its structure. There has been some work involving $\mathrm{SO}(10,2)$ models that use the specialized superalgebra $S_{\alpha \beta}=\gamma_{\alpha \beta}^{\mu \nu}\left(p_{\mu} v_{\nu}-p_{\nu} v_{\mu}\right)$ suggested in [16]. These are super-Yang-Mills type fields [23] and strings [24] that fit into the $S$-theory framework. However, these constructions were restricted to a single eigenvalue of $v_{\mu}$, and therefore they break the $\operatorname{SO}(10,2)$ symmetry. Their generalizations in the sense of Eq. (54) would remove this restriction.

(6) It would be interesting to try to understand the $\mathrm{SO}(c+1,1)$ symmetry discussed here from the point of view of $M$ and $F$ theories [17-20].

\section{ACKNOWLEDGMENTS}

This research was supported by the U.S. Department of Energy under Grant No. DE-FG03-84ER40168.
[1] A. Strominger and C. Vafa, Phys. Lett. B 379, 104 (1996); J. Breckenridge, D. Lowe, R. Myers, A. Peet, A. Strominger, and C. Vafa, ibid. 381, 423 (1996).

[2] G. Horowitz and A. Strominger, Phys. Rev. Lett. 77, 2363 (1996); G. Horowitz, J. Maldacena, and A. Strominger, Phys. Lett. B 383, 151 (1996).

[3] G. Horowitz, "The origin of black hole entropy in string theory,', Report No. gr-qc/9604051 (unpublished).

[4] M. Cvetic and D. Youm, Phys. Rev. D 54, 2612 (1996); Nucl. Phys. B477, 449 (1996); B476, 118 (1996).

[5] M. Cvetic and A. A. Tseytlin, Phys. Lett. B 366, 95 (1996); Phys. Rev. D 53, 5619 (1996).
[6] E. Halyo, B. Kol, A. Rajaraman, and L. Susskind, "Counting Schwarzchild and charged black holes,' Report No. hep-th/ 9609075 (unpublished).

[7] R. Dijkgraaf, E. Verlinde, and H. Verlinde, "BPS spectrum of the five brane and black hole entropy,' Report No. hep-th/ 9603126 (unpublished); "BPS quantization of the five brane,', Report No. hep-th/9604055 (unpublished).

[8] R. Kallosh and B. Kol, Phys. Rev. D 53, 5344 (1996).

[9] S. Ferrara and R. Kallosh, Phys. Rev. D 54, 1514 (1996); 54, 1525 (1996).

[10] M. Cvetic and C. Hull, Nucl. Phys. B480, 296 (1996).

[11] K. Behrndt, R. Kallosh, J. Rahmfeld, M. Shmakova, and W. 
Kai Wong, Phys. Rev. D 54, 6293 (1996).

[12] R. Kallosh and A. Rajaraman, Phys. Rev. D 54, 6381 (1996).

[13] J. Polchinski, Phys. Rev. Lett. 75, 4724 (1995); See also J. Polchinski, S. Chaudhuri, and C. V. Johnson, "Notes on D-branes," Report No. hep-th/9602052 (unpublished).

[14] C. Hull and P. Townsend, Nucl. Phys. B438, 109 (1995).

[15] I. Bars, Phys. Rev. D 54, 5203 (1996); in Proceedings of Frontiers in Quantum Field Theory, Toyonaka, Japan, 1996 (unpublished), Report No. hep-th/9604200 (unpublished).

[16] I. Bars, Phys. Rev. D 55, 2373 (1997).

[17] E. Witten, Nucl. Phys. B443, 85 (1995); "Some comments on String Dynamics," Report No. hep-th/9507121, in the Proceedings of Strings '95 (in press); P. Horava and E. Witten, Nucl. Phys. B460, 506 (1996).

[18] P. Townsend, “p-brane democracy,' Report No. hep-th/ 9507048 (unpublished).

[19] J. Schwarz, Phys. Lett. B 367, 97 (1996); in Theory of Elementary Particles, Proceedings of the 29th International Ahren- shoop Symposium, Buckow, Germany, 1995, edited by D. Lust and G. Weigt [Nucl. Phys. B (Proc. Suppl.) 49 (1996)]; Report No. hep-th/9509148 (unpublished), “M-theory extensions of T duality,' Report No. hep-th/9601077 (unpublished).

[20] C. Vafa, Nucl. Phys. B469, 403 (1996); D. R. Morrison and C. Vafa, ibid. B473, 74 (1996); B476, 437 (1996); E. Witten, ibid. B471, 195 (1996).

[21] I. Bars, Phys. Rev. D 52, 3567 (1995); I. Bars and S. Yankielowicz, ibid. 53, 4489 (1996); I. Bars, in Theory of Elementary Particles [19], p. 115.

[22] E. Cremmer, "On hidden symmetries in extended supergravities," Report No. LPTENS 83/1, 1983 (unpublished); presented at the September School on Supergravity and Supersymmetry, Trieste, Italy, 1982, Trieste Workshop 1982, Report No. QC178:T7:1982 (unpublished), p. 153.

[23] H. Nishino and E. Sezgin, Phys. Lett. B 388, 569 (1996).

[24] E. Martinec, "Geometrical structures of M-theory,', Report No. hep-th/9608017 (unpublished). 